\title{
Efeito da exploração madeireira sobre o número de indivíduos férteis de três espécies arbóreas comerciais na Amazônia oriental
}

\author{
Marisa Gesteira FONSECA ${ }^{1}$, Edson VIDAL ${ }^{2}$, Flavio Antonio Maës dos SANTOS 3 \\ RESUMO \\ Estudos da ecologia reprodutiva de árvores são fundamentais para compreender os possíveis impactos da exploração madeireira \\ e para subsidiar o aperfeiçoamento das práticas de manejo. Os objetivos desse trabalho foram: 1) estimar a proporção e o \\ número de indivíduos reprodutivos por classe de diâmetro de Chrysophyllum lucentifolium subsp. pachycarpum, Lecythis lurida \\ e Pseudopiptadenia psilostachya, três espécies madeireiras, em uma floresta em Paragominas (PA) e; 2) estimar o impacto da \\ exploração de $90 \%$ dos indivíduos com DAP $\geq 50 \mathrm{~cm}$ sobre o número de indivíduos reprodutivos das mesmas espécies no \\ local. Durante uma estação reprodutiva de cada espécie, foram amostradas 80 árvores de L. lurida, 76 de $P$. psilostachya e 76 \\ de C. lucentifolium. Foi estimado que 14,9\% de todos os indivíduos férteis de C. lucentifolium, 35,9\% de L. lurida e 72,4\% \\ de $P$. psilostachya tinham DAP $\geq 50 \mathrm{~cm}$ no ano de amostragem. Assim, o corte de $90 \%$ dessas árvores causaria uma redução \\ de 13,4\%, 32,6 \% e 65,2 \% do número de indivíduos férteis de C. lucentifolium, L. lurida e P. psilostachya, respectivamente. \\ Se as proporções de indivíduos férteis fossem constantes ao longo do tempo, para preservar metade dos indivíduos férteis de \\ P. psilostachya seria necessário manter $30 \%$ e não $10 \%$ daqueles com DAP $\geq 50 \mathrm{~cm}$. Os resultados indicam que, adotando-se \\ um único diâmetro mínimo de corte e retendo-se a mesma proporção de árvores acima desse diâmetro, o efeito em termos \\ de porcentagem de indivíduos reprodutivos retirados da população pode variar entre espécies na ordem de aproximadamente \\ cinco vezes.
}

PALAVRAS-CHAVE: Diâmetro mínimo de corte, Ecologia reprodutiva, Exploração madeireira, Frutificação.

\section{Effects of logging on the number of fertile individuals of three commercial tree species in Eastern Amazonia}

\begin{abstract}
Studies concerning the reproductive ecology of trees are important so as to better understand the impacts of logging and for the definition of guidelines to improve management practices. The aim of this study was to estimate (1) the number and proportion of reproductive individuals of Chrysophyllum lucentifolium subsp. pachycarpum, Lecythis lurida e Pseudopiptadenia psilostachya per size class, and (2) the impact of harvesting $90 \%$ of individuals $\geq 50 \mathrm{~cm}$ dbh on the number of reproductive trees. We sampled $80 \mathrm{~L}$. lurida, $76 \mathrm{P}$. psilostachya and $76 \mathrm{C}$. Iucentifolium trees during one reproductive season. We estimated that $14.9 \%$ of all fertile individuals of $C$. lucentifolium, $35.9 \%$ of L. lurida and $72.4 \%$ of $P$. psilostachya were $\geq 50 \mathrm{~cm}$ dbh in that year. Therefore, the harvest of $90 \%$ of these trees would cause a $13.4 \%, 32.6 \%$, and $65.2 \%$ reduction in the number of fertile individuals of $C$. lucentifolium, L. lurida and $P$. psilostachya, respectively. If these proportions were stable over time, it would be necessary to keep $30 \%$ instead of $10 \%$ of individuals $\geq 50 \mathrm{~cm} \mathrm{dbh}$ in order to preserve half of the $P$. psilostachya fertile individuals. The results indicate that the effects of adopting a single minimum cutting diameter and of setting aside the same proportion of trees above that diameter on the proportion of remaining fertile individuals may vary by a factor of five among species.
\end{abstract}

KEYWORDS: Logging, Minimum cutting diameter, Reproductive ecology.

\footnotetext{
1 UNICAMP. Programa de Pós-graduação em Biologia Vegetal, Departamento de Botânica, Instituto de Biologia, CP 6109, Universidade Estadual de Campinas, CEP 13083-970, Campinas, SP, Brasil. e-mail: marisa_fonseca@yahoo.com.br

2 Esalq/USP. Departamento de Ciências Florestais, ESALQ/USP, Caixa Postal 9, 13418-900, Piracicaba, SP, Brasil. e-mail: edvidal@esalq.usp.br

${ }^{3}$ UNICAMP. Departamento de Botânica, Instituto de Biologia, CP 6109, Universidade Estadual de Campinas, 13083-970, Campinas, SP, Brasil. e-mail: fsantos@unicamp.br
} 


\section{INTRODUÇÃO}

A exploração madeireira seletiva em florestas naturais implica na retirada de indivíduos reprodutivos das espécies arbóreas comerciais, podendo reduzir significativamente a produção total de frutos e sementes das populações exploradas (Gullison et al., 1996; Grogan \& Galvão, 2006). Tendo em vista que a regeneração de árvores tropicais é limitada pela disponibilidade de sementes (Svenning \& Wright, 2005), tal redução pode afetar a regeneração de espécies comerciais (Plumptre, 1995), assim como a quantidade de recursos alimentares para a fauna (Johns, 1988). Estudos da ecologia reprodutiva e regeneração de árvores são fundamentais tanto para compreender os possíveis impactos da exploração madeireira quanto para subsidiar o aperfeiçoamento das práticas de manejo (Guariguata \& Pinard, 1998; Sheil \& Heist, 2000).

Segundo a legislação brasileira que define as práticas de exploração madeireira em florestas naturais na Amazônia (Brasil, 2006), o diâmetro mínimo de corte (DMC) de cada espécie deve ser definido com base em estudos técnicos e, na ausência destes, poderão ser cortadas árvores com diâmetro à altura do peito (DAP) igual ou superior a $50 \mathrm{~cm}$. Entre outras práticas, a Instrução Normativa determina ainda que pode ser explorado até $90 \%$ dos indivíduos acima do DMC de uma espécie na área destinada ao manejo em um dado ano, podendo essa porcentagem ser reduzida ou aumentada de acordo com a distribuição diamétrica e outras características ecológicas da espécie em questão. Tais informaçōes, contudo, não estão disponíveis para as espécies madeireiras da Amazônia brasileira, com raras exceçōes (Grogan e Galvão, 2006; Grogan et al, 2006; Lacerda, 2007).

Chrysophyllum lucentifolium subsp. pachycarpum Pires e T.D. Penn. (Sapotaceae), Lecythis lurida (Miers) Mori (Lecythidaceae) e Pseudopiptadenia psilostachya (DC.) G.P. Lewis \& M.P. Lima (Leguminosae) são espécies arbóreas comerciais de grande porte (dossel superior ou emergentes) e alta densidade de indivíduos na floresta onde foi realizado o presente estudo. Suas madeiras podem ser utilizadas na construção civil externa e interna, entre outras aplicações (Sudam, 1981; Teixeira et al., 1988; Loureiro et al., 2000).

Os objetivos desse trabalho foram: 1) estimar a proporção e o número de indivíduos reprodutivos por classe de tamanho dessas três espécies em uma floresta em Paragominas (PA) e; 2) estimar o impacto da exploração de $90 \%$ dos indivíduos com $\mathrm{DAP} \geq 50 \mathrm{~cm}$ sobre o número de indivíduos reprodutivos das mesmas espécies no local.

\section{MATERIAL E MÉTODOS}

Esse estudo foi realizado em uma floresta de terra firme na Fazenda Agrosete $\left(3^{\circ} 00^{\prime}-3^{\circ} 05^{\prime} \mathrm{S}\right.$; $\left.47^{\circ} 10^{\prime}-47^{\circ} 15^{\prime} \mathrm{W}\right)$,
Paragominas, PA. O dossel na área atinge de 25 a $40 \mathrm{~m}$ de altura e a densidade média é igual a 206 indivíduos entre 15 $\mathrm{cm}$ e $45 \mathrm{~cm}$ de DAP e 17 indivíduos com DAP $\geq 45 \mathrm{~cm}$ por ha (Barreto et al., 1998). Os solos da região são latossolos e a precipitação anual média é de $1750 \mathrm{~mm}$, com uma estação seca bem definida ( $<50 \mathrm{~mm}$ por mês) de julho a novembro (Holmes et al., 2002). O relevo é relativamente plano, com inclinação de no máximo $5^{\circ}$ (Barreto et al., 1998). As árvores foram amostradas em três áreas de 24,5 ha cada. Duas delas foram submetidas à exploração madeireira em 1993 (com e sem planejamento prévio, veja Johns et al. (1996) para uma descrição detalhada das práticas adotadas) e na terceira área não há registro de perturbação recente.

C. lucentifolium é popularmente conhecida no Brasil como abiu-casca-grossa, abiu-branco, abiurana, abiurana-goiaba, cariúba, guajará ou goiabão. Seus frutos são drupas ovóides, subglobosas ou obovóides, com látex branco (Pennington, 1990) e constituem importante fonte de alimento para aves, macacos, pacas, cotias, porcos e veados (Feer \& Forget, 2002). Sua distribuição geográfica inclui o Panamá, Equador, Peru, Colômbia, Venezuela, Guiana Francesa e Amazônia brasileira (Pennington, 1990). Normalmente ocorre em florestas úmidas abaixo de $500 \mathrm{~m}$ de altitude, mas é também encontrada em florestas montanas de até $1400 \mathrm{~m}$ de altitude na Venezuela e em regiōes secas no Equador e no Peru (Pennington, 1990).

L. lurida é uma espécie decídua, conhecida popularmente como jarana, jarana-branca, jarana-buroja, inhaúba, inhaíba ou inhaíba-gigante. Os frutos são pixídios indeiscentes, com pericarpo coriáceo (Mori \& Prance, 1990). Ocorre ao longo da costa brasileira do Rio de Janeiro a Pernambuco e na amazônia oriental, em florestas maduras não sujeitas a alagamento, florestas secundárias e cerrados, apresentando menor estatura nesses dois últimos ambientes (Mori \& Prance, 1990).

P. psilostachya é chamada popularmente de timborana, caferana-açu ou faveira-folha-fina. Os frutos são longas vagens deiscentes com até $60 \mathrm{~cm}$ de comprimento (Parrota et al., 1995). É típica da região amazônica, sendo encontrada no Brasil em florestas de terra firme do Pará, Amazonas, Amapá, Roraima e Rondônia. Ocorre também na Guiana Francesa, Suriname, Guiana, Venezuela e Colômbia (Lewis \& Lima, 1990).

Para a estimativa visual da frutificação, foram sorteados, em cada uma das três áreas, cinco indivíduos de cada espécie em cada uma das seis classes de DAP $(10$ a $20 \mathrm{~cm}, 20$ a 30 $\mathrm{cm}, 30$ a $40 \mathrm{~cm}, 40$ a $50 \mathrm{~cm}, 50$ a $60 \mathrm{~cm},>60 \mathrm{~cm})$. Os dados de inventário utilizados para o sorteio de indivíduos foram coletados em 2000, mas nas análises foram considerados os valores de DAP em 2003. Três indivíduos de C. lucentifolium mudaram de classe de tamanho entre 2000 e 2003, o que explica a amostragem de 18 árvores dessa espécie com $40 \mathrm{~cm}$ $\leq \mathrm{DAP}<50 \mathrm{~cm}$. Em algumas classes de tamanho de algumas 
espécies existiam menos de cinco indivíduos disponíveis por tratamento e nesses casos todos os indivíduos disponíveis na classe foram amostrados. Para C. lucentifolium, as duas últimas classes de tamanho foram agrupadas devido ao baixo número de indivíduos.

Durante uma estação reprodutiva de cada espécie, a intensidade de frutificação de indivíduos foi avaliada visualmente com o uso de binóculos em intervalos de aproximadamente 15 dias até que os últimos frutos fossem observados nas copas, sendo classificada como alta (copa completa ou abundantemente coberta de frutos), baixa (poucos frutos em um ou poucos galhos) ou média (intermediária entre baixo e alto; Chapman \& Chapman, 1997; Lepsch-Cunha \& Mori, 1999).

Os primeiros frutos de C. lucentifolium em estádio inicial de desenvolvimento foram observados na primeira quinzena de setembro de 2003. A estimativa visual da frutificação dessa espécie foi iniciada na segunda quinzena de outubro de 2003 e se estendeu até a segunda quinzena de fevereiro de 2004. Em novembro e início de dezembro de 2003 foram vistas árvores de L. lurida com flores e os primeiros frutos imaturos foram avistados na segunda quinzena de janeiro de 2004. Indivíduos dessa espécie começaram a ser vistoriados a partir do início da segunda quinzena de fevereiro e a estimativa visual se estendeu até a primeira quinzena de abril de 2004 . Indivíduos de $P$. psilostachya floresceram em setembro e outubro de 2003 e os primeiros frutos em estádio inicial de desenvolvimento foram vistos na segunda quinzena de novembro do mesmo ano. Árvores dessa espécie foram visitadas entre o início da segunda quinzena de dezembro de 2003 e da primeira quinzena de abril de 2004. A frutificação foi avaliada em 80 árvores de $L$. lurida, 76 de $P$. psilostachya e 76 de C. lucentifolium.

Foi estimada, dessa forma, a proporção de árvores reprodutivas por classe de tamanho no ano de estudo. A partir de um inventário realizado em 2003 de todas as árvores com $\mathrm{DAP} \geq 10 \mathrm{~cm}$ na área não explorada, foi possível estimar o número e a distribuição de tamanho de todos os indivíduos férteis das espécies estudadas nessa floresta. A proporção estimada de indivíduos férteis com $20 \mathrm{~cm} \leq \mathrm{DAP}<50 \mathrm{~cm}$ e com DAP $\geq 50 \mathrm{~cm}$ foi comparada entre espécies através de teste de Qui-quadrado de partição (Ayres et al. 2003). Supondo-se que a intensidade de exploração (em termos de porcentagem de indivíduos cortados) seja a mesma para indivíduos férteis e estéreis acima do diâmetro mínimo de corte, foi estimado o efeito do abate de $90 \%$ das árvores com DAP $\geq 50 \mathrm{~cm}$ sobre o número de indivíduos férteis na floresta primária.

\section{RESULTADOS E DISCUSSÃO}

Aproximadamente $47 \%$ dos indivíduos de C. lucentifolium acompanhados durante a estação reprodutiva produziram frutos e a menor árvore que frutificou possuía $22,8 \mathrm{~cm}$ de DAP. A proporção de árvores férteis dessa espécie foi aproximadamente 2 vezes maior a partir de $40 \mathrm{~cm}$ de DAP do que na classe de $30 \mathrm{~cm}$ a $40 \mathrm{~cm}$ de DAP (Figura 1). A proporção de árvores de $L$. lurida férteis entre aquelas acompanhadas foi de $-41 \%$ e a porcentagem de indivíduos reprodutivos por classe de tamanho aumentou a partir de 30 $\mathrm{cm}$ de DAP (Figura 1). A menor árvore dessa espécie que frutificou tinha 20,0 cm de DAP. Apenas 15 indivíduos de P. psilostachya (20\% do total amostrado) produziram frutos e a menor árvore que frutificou possuía $29,0 \mathrm{~cm}$ de DAP. A proporção de árvores férteis foi mais de 2,5 vezes maior entre
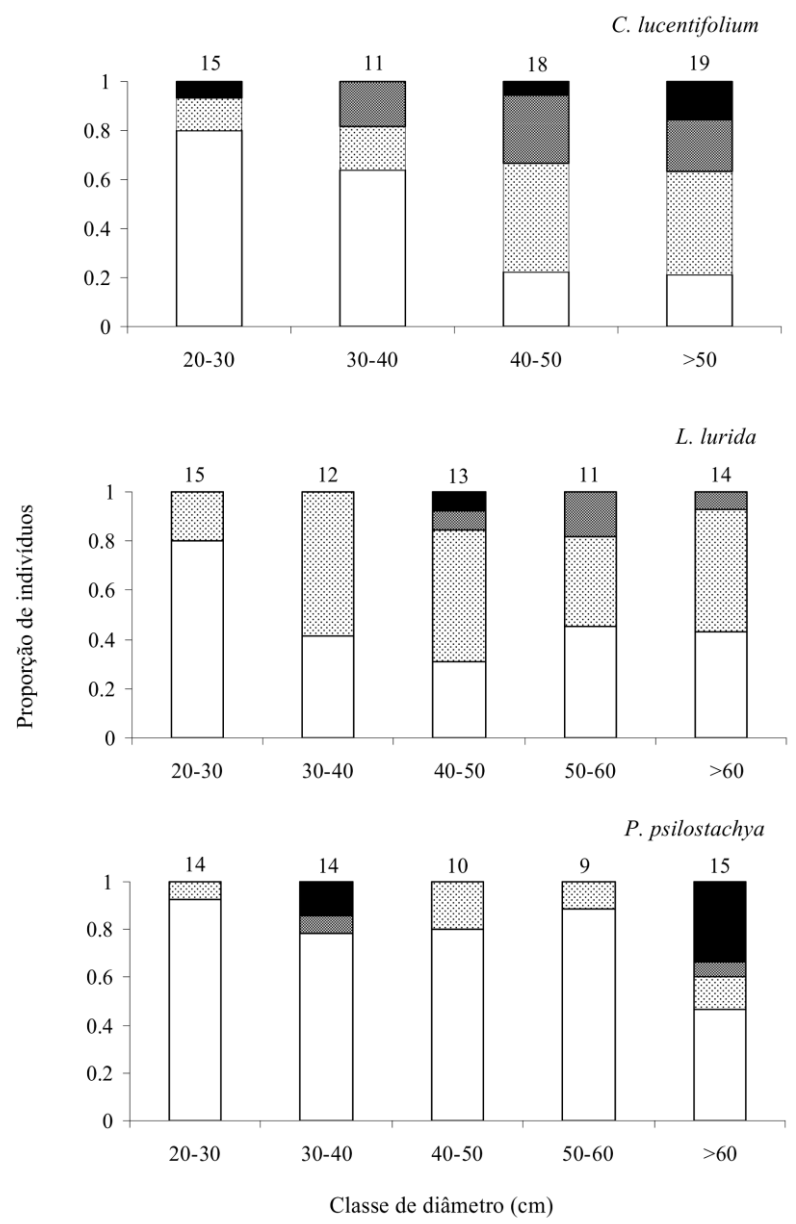

$\square$ sem fruto $⿴ 囗 大)$ frutificação baixa $\square$ frutificação intermediária $\square$ frutificação alta

Figura 1 - Proporção de árvores classificadas em diferentes categorias de frutificação em cada classe de diâmetro a altura do peito das três espécies estudadas. No caso de C. lucenfolium as duas últimas classes de tamanho foram agrupadas devido ao baixo número de indivíduos. Valores acima das barras se referem ao número total de indivíduos em cada classe de diâmetro. Nenhum indivíduo $10 \mathrm{~cm} \leq \mathrm{DAP}<20 \mathrm{~cm}$ produziu frutos. 
as árvores com DAP $\geq 60 \mathrm{~cm}$ do que nas demais classes de tamanho (Figura 1).

Entre todos os indivíduos de C. lucentifolium com DAP $\geq$ $20 \mathrm{~cm}$ na floresta primária estudada $(\mathrm{N}=94)$, apenas $-9,6 \%$ tinham DAP $\geq 50 \mathrm{~cm}$. Essa proporção foi $-3,4$ vezes maior em L. lurida $(\mathrm{N}=31$ indivíduos com $\mathrm{DAP} \geq 20 ; 32,3 \%$ dos quais com $\mathrm{DAP} \geq 50 \mathrm{~cm}) \mathrm{e}-5,5$ vezes maior em $P$. psilostachya $(\mathrm{N}=59,52,5 \%$ dos quais com $\mathrm{DAP} \geq 50 \mathrm{~cm})$. Aplicando-se a proporção de indivíduos férteis ao número total de indivíduos em cada classe de tamanho na floresta primária, estima-se que, nessa área, $14,9 \%$ de todos os indivíduos férteis de $C$. lucentifolium, 35,9\% de L. lurida e 72,4\% de P. psilostachya tinham DAP $\geq 50 \mathrm{~cm}$ no ano de amostragem (Figura 2). A proporção estimada de indivíduos férteis com $20 \mathrm{~cm} \leq \mathrm{DAP}$ $<50 \mathrm{~cm}$ e com DAP $\geq 50 \mathrm{~cm}$ não diferiu significativamente entre $C$. lucentifolium e L. lurida $\left(\chi_{1}^{2}=2,446 ; \mathrm{p}=0,117\right)$, mas sim entre essas e $P$. psilostachya $\left(\chi_{1}^{2}=16,498 ; \mathrm{p}<0,001\right)$.

O corte de $90 \%$ das árvores com DAP $\geq 50 \mathrm{~cm}$ implicaria em uma redução de 13,4\%, 32,6 \% e 65,2 \% do número de indivíduos férteis de C. lucentifolium, L. lurida e P. psilostachya, respectivamente. Como a proporção de indivíduos classificados nas categorias de frutificação intermediária e alta é maior nas maiores classes de tamanho (Figura 1), estima-se de forma análoga que, respectivamente, $14,4 \%, 48,0 \%$ e 70,0 \% dos indivíduos de C. lucentifolium, L. lurida e P. psilostachya nessas categorias de frutificação seriam cortados se fosse adotada a prática de manejo acima mencionada.

Se as proporções de indivíduos férteis fossem constantes ao longo do tempo, para preservar metade dos indivíduos férteis de $P$. psilostachya seria necessário manter $30 \%$ e não $10 \%$ daqueles com DAP $\geq 50 \mathrm{~cm}$. De forma similar, Lacerda (2007) estimou que aproximadamente 65\% dos indivíduos reprodutivos de Jatobá (Hymenaea courbaril) seriam cortados em um plano de manejo executado na Floresta Nacional de Tapajós (PA) que previa a derrubada de todos os indivíduos

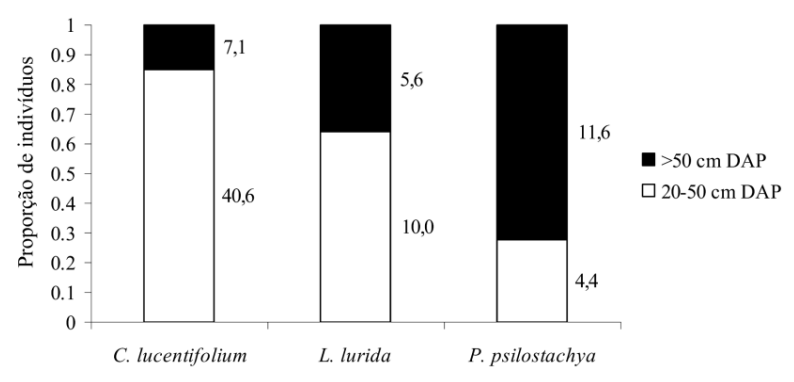

Figura 2 - Estimativa da proporção dos indivíduos férteis das três espécies estudadas com diâmetro a altura do peito (DAP) menor e maior que $50 \mathrm{~cm}$ em 24,5 ha de floresta primária em Paragominas (PA). Valores ao lado das colunas correspondem ao número estimado de indivíduos em cada categoria de tamanho. da espécie com DAP $\geq 81 \mathrm{~cm}$ (equivalente a $54 \%$ de todos com $\mathrm{DAP} \geq 50 \mathrm{~cm}$ ).

Os resultados obtidos no presente estudo indicam que, adotando-se um único $\mathrm{DMC}(\mathrm{DAP} \geq 50 \mathrm{~cm})$ e retendo-se a mesma proporção (10\%) de árvores acima desse diâmetro, o efeito em termos de porcentagem de indivíduos reprodutivos retirados da população pode variar entre espécies na ordem de aproximadamente cinco vezes. Essa variação interespecífica se deve às diferenças tanto na distribuição de indivíduos por classe de tamanho quanto na proporção de indivíduos férteis em cada classe.

Adicionalmente, foi observado que o tamanho mínimo em que as árvores começam a se reproduzir não é um bom indicativo do efeito das práticas de manejo. No caso de $P$. psilostachya, por exemplo, apesar de árvores com DAP $\geq 29 \mathrm{~cm}$ serem capazes de produzir frutos, a proporção de indivíduos férteis só aumenta a partir de $60 \mathrm{~cm}$ de DAP.

A frutificação de espécies arbóreas é amplamente variável entre anos (e.g. De Steven \& Wright, 2002; Kelly \& Sork, 2002; Pedroni et al., 2002) e a continuidade do acompanhamento das espécies estudadas é necessária para uma avaliação mais consistente dos efeitos da exploração madeireira sobre a produção de frutos de cada população. Tendo em vista que nem todos os indivíduos férteis se reproduzem todo ano, a avaliação realizada em um único ano possivelmente implica em uma subestimativa da redução do número destes indivíduos. Além disso, o efeito da exploração deve ser maior se forem consideradas estimativas quantitativas da intensidade de frutificação, já que indivíduos maiores tendem a produzir uma maior quantidade de frutos (Chapman et al., 1992; Grogan \& Galvão, 2006). Estudos sobre o sistema reprodutivo e a polinização de tais espécies também são de fundamental importância para a avaliação do impacto da exploração, já que o aumento da distância entre indivíduos férteis pode afetar a atividade dos polinizadores e, conseqüentemente, a qualidade e quantidade de sementes produzidas pelos indivíduos remanescentes (Sheil \& Heist, 2000; Ghazoul \& McLeish, 2001).

Apesar de se referir apenas à proporção de indivíduos férteis em um único evento reprodutivo, o presente estudo sugere que a produção de frutos de algumas espécies madeireiras pode ser intensamente afetada pelas práticas de exploração atualmente permitidas pela legislação brasileira em florestas naturais da Amazônia, caso tais práticas não sejam adaptadas para as características reprodutivas de cada espécie. É urgente, dessa forma, a realização de estudos sobre a ecologia reprodutiva de árvores madeireiras, assim como do efeito da retirada dos maiores indivíduos sobre a dinâmica populacional das mesmas (Gourlet-Fleury et al., 2005; Fonseca, 2007; Lacerda, 2007). Critérios para a escolha de espécies a serem estudadas podem incluir a intensidade de exploração à qual a espécie 
está sujeita, sua importância para a fauna e/ou para a estrutura florestal e a susceptibilidade potencial aos efeitos da exploração (avaliada através de critérios como distribuição geográfica, síndrome de dispersão das sementes, taxa de crescimento, entre outros; Martini et al., 1994). Tais estudos devem subsidiar o aperfeiçoamento das práticas de manejo e favorecer, portanto, a sustentabilidade do manejo florestal.

\section{AGRADECIMENTOS}

Esse estudo foi financiado pela Fundação de Apoio à Pesquisa do Estado de São Paulo (FAPESP; processo 02/14073-5), pela WWF Brasil (processo CSR 3042004) e pelo Conselho Nacional de Desenvolvimento Científico e Tecnológico (CNPq) através do Edital Universal (processo 472962/2003-5), da bolsa de doutorado cedida à primeira autora (processo 141465/2002-6) e da bolsa de produtividade em pesquisa cedida ao terceiro autor (processo 304937/2007-0). Os autores são gratos ainda ao Imazon (Instituto do Homem e Meio Ambiente da Amazônia) pelo apoio logístico e aos proprietários da Fazenda Agrosete, Pérsio e Tales Lima, pela permissão para a coleta de dados na área.

\section{BIBLIOGRAFIA CITADA}

Ayres, M.; Ayres Jr, M.; Ayres, D.L.; Santos, A.L. 2003. BioEstat 3.0: aplicaçôes estatísticas nas áreas das ciências biológicas e médicas. Sociedade Civil Mamirauá, Belém, Pará. 290pp.

Barreto, P.; Amaral, P.; Vidal, E.; Uhl, C. 1998. Costs and benefits of forest management for timber production in eastern Amazonia. Forest Ecology and Management, 108: 9-26.

Brasil. 2006. Ministério do Meio Ambiente, Instrução Normativa no 5, de 11 de Dezembro de 2006. Dispóe sobre os procedimentos técnicos para elaboração, apresentação, execução e avaliação técnica de Planos de Manejo Florestal Sustentável - PMFSs nas florestas primitivas na Amazônia legal. Disponível em: http:// www.mma.gov.br/estruturas/sfb/_arquivos/in05_manejo_ florestal.pdf. Acesso em 02 de julho de 2007.

Chapman, C.A.; Chapman, L.J.; Wanghan, R.; Hunt, K.; Gebo, D.; Gardner, L. 1992. Estimators of fruit abundance of tropical trees. Biotropica, 24: 527-531.

Chapmam, C.A; Chapman L.J. 1997. Forest regeneration in logged and unlogged forests of Kibale National Park, Uganda. Biotropica, 29:396 - 412.

De Steven, D.; Wright, S.J. 2002. Consequences of variable reproduction for seedling recruitment in three neotropical tree species. Ecology, 83: 2315-2327.

Feer, F; Forget, P.M. 2002. Spatio-temporal variation in postdispersal seed fate. Biotropica, 34: 555-566.

Fonseca, M.G. 2007. Ecologia populacional de três espécies madeireiras na Amazônia oriental: implicaçóes para o manejo. Tese de Doutorado. Universidade Estadual de Campinas, Campinas, São Paulo. 152pp.
Ghazoul, J.; Mc Leish, M. 2001. Reproductive ecology of tropical forest trees in logged and fragmented habitats in Thailand and Costa Rica. Plant Ecology, 153: 335-345.

Gourlet-Fleury, S.; Cornu, G.; Jésel ,S.; Dessard, H.; Jourget, J.; Blanc, L.; Picard, N. 2005. Using models to predict recovery and assess tree species vulnerability in logged tropical forests: A case study from French Guiana. Forest Ecology and Management, 209: 69-85.

Grogan, J.E.; Galvão, J. 2006. Factors limiting post-logging seedling regeneration by bigleaf mahogany (Swietenia macrophylla) in Southeastern Amazonia, Brazil, and implications for sustainable management. Biotropica, 38:219-228.

Grogan, J.; Vidal, E.; Schulze, M. 2006. Apoio científico para os padrões de manejo de madeira na floresta Amazônica: a questão da sustentabilidade. Ciência e Ambiente, 32: 103-117.

Guariguata, M.R.; Pinard, M.A. 1998. Ecological knowledge of regeneration from seed in neotropical forest trees: implication for natural forest management. Forest Ecology and Management, 112: 87-99.

Gullison, R.E; Panfil, S.N.; Strouse, J.J.; Hubbell, S.P. 1996. Ecology and management of mahogany (Swietenia macrophylla King) in the Chimanes forest, Beni, Bolivia. Botanical Journal of the Linnean Society, 122: 9-34.

Holmes, T.P.; Blate, G.M.; Zweede, J.C.; Pereira Jr., R.; Barreto, P.; Boltz, F.; Bauch, R. 2002. Financial and ecological indicators of reduced impact logging performance in the eastern Amazon. Forest Ecology and Management, 163: 93-110.

Johns, A.D. 1988. Effects of "selective" timber extraction on rain forest structure and composition and some consequences for frugivores and folivores. Biotropica, 20: 31-37.

Johns, J.; Barreto, P.; Uhl, C. 1996. Logging damage in planned and unplanned logging operations and its implications for sustainable timber production in eastern Amazon. Forest Ecology and Management, 89: 59-77.

Kelly, D.; Sork, V.L. 2002. Mast seeding in perennial plants: Why? How? Where? Annual Review of Ecology and Systematics, 33:427- 447.

Lacerda, A.E.B. 2007. Ecological and genetic impacts of reducedimpact logging in the Brazilian Amazonian forest: the case of Hymenaea courbaril L. Tese de Doutorado. The University of Reading, Reading, UK. 310pp.

Lepsch-Cunha, N.; Mori, S.A. 1999. Reproductive phenology and mating potential in a low density tree population of Couratari multiflora (Lecythidaceae) in central Amazonia. Journal of Tropical Ecology, 15: 97-121.

Lewis, G.P.; Lima, M.P.M. 1990. Pseudopiptadenia Rauschert no Brasil (Leguminosae, Mimosoideae). Arquivos do Jardim Botânico do Rio de Janeiro, 30: 43-67.

Loureiro, A.A.; Freitas, J.A.; Ramos, K.B.L.; Freitas, C.A.A. 2000. Essências madeireiras da Amazônia. MCT/INPA, Manaus, Amazonas.191pp.

Martini, A.M.Z.; Rosa, N.A.; UHL, C. 1994. An attempt to predict which amazonian tree species may be threatened by logging activities. Environmental Conservation, 21: 152-162. 
Mori, S.A.; Prance, G.T. 1990. Flora Neotropica: Lecythidaceae Part II. The New York Botanical Garden, New York, NY. 384pp.

Parrota, J.A.; Francis, J. K.; Almeida, R.R. 1995. Trees of the Tapajos: a photographic field guide. USDA Forest Service, Río Piedras, Puerto Rico. 370pp.

Pedroni, F.; Sanchoez, M.; Santos, F.A.M. 2002. Fenologia da copaíba (Copaifera langsdorffi $i$ Desf. Leguminosae, Caesalpinioideae) em uma floresta semidecídua no sudeste do Brasil. Revista Brasileira de Botânica, 25: 183-194.

Pennington, T.D. 1990. Flora Neotropica: Sapotaceae. The New York Botanical Garden, New York, NY. 770pp.

Plumptre, A.J. 1995 The importance of "seed trees" for the natural regeneration of selectively logged tropical forest. Commonwealth Forestry Review, 74: 253-258.
Sheil, D.; Heist, M.V. 2000. Ecology for tropical forest management. International Forestry Review, 2: 261-270.

Sudam 1981. Grupamento de espécies tropicais da Amazônia por similaridade de características básicas e por utilização. Sudam, Belém, Pará.237pp.

Svenning, J.C.; Wright, S.J. 2005. Seed limitation in a Panamanian forest. Journal of Ecology, 93: 853-862.

Teixeira, D.E.; Santana, M.A.E.; Souza, M.R. 1988. Amazonian timbers for the international market. IBDF/ITTO, Brasília, D.F. 94pp.

Recebido em 12/09/2007

Aceito em 21/05/2008 